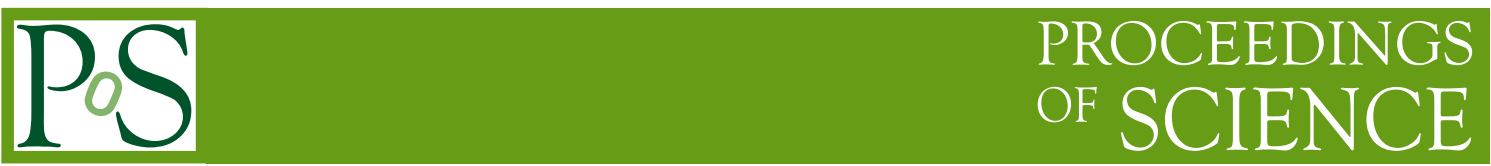

\title{
Single-top quark studies in ATLAS
}

\author{
Dominic Hirschbühl on behalf of the ATLAS Collaboration* \\ Bergische Universität Wuppertal \\ E-mail: dominic.hirschbuehlecern.ch
}

\begin{abstract}
We present the result of searches for single top-quark production in the $t$ and $W t$-channels in 7 $\mathrm{TeV}$ proton-proton collisions with the ATLAS detector using $0.7 \mathrm{fb}^{-1}$ integrated luminosity. The $t$-channel search is based on the selection of events with a single lepton (muon or electron), jets and missing transverse energy. The $W t$-channel analysis is based on the selection of events with two leptons, jets and missing transverse energy.

The $t$-channel production cross section is measured by two different techniques, one following a cut-based approach and one fitting the distribution of a multivariate discriminant constructed with a neural network. The cut-based analysis combines the 2-jet and 3-jet data sets and measures $\sigma_{t}=90_{-22}^{+32} \mathrm{pb}$. The observed significance is 7.6 standard deviations.

A simple cut-based approach is used to select the $W t$ contribution. After selection, we observe 287 data events compared to a prediction of 293 events, with a predicted signal purity of $16 \%$. The following upper limits at 95\% CL are determined on the $W t$ associated production cross-section : $\sigma(p p \rightarrow W t+X)<39(41) \mathrm{pb}$ for the observed (expected) limit.
\end{abstract}

The 2011 Europhysics Conference on High Energy Physics-HEP 2011,

July 21-27, 2011

Grenoble, Rhône-Alpes France

* Speaker. 


\section{Introduction}

At the LHC, single top-quarks can be produced at tree level in three processes. The $t$-channel exchange of a virtual $W$ boson has a predicted production cross section of $64.6 \mathrm{pb}$ [1]. The associated production of an on-shell $W$ boson and a top quark ( $W t$-channel), which has a predicted production cross section of $15.7 \mathrm{pb}$ [2]. The $s$-channel production and decay of a virtual $W$ boson has a predicted production cross section of $4.6 \mathrm{pb}$ [3]. The single top-quark final state provides a direct probe of the $W t b$ coupling and thus determines $\mathrm{V}_{\mathrm{tb}}$ without assumptions about the number of quark generations and is sensitive to many models of new physics [4]. Alternatively, it allows the $b$-quark parton distribution function (PDF) to be measured.

This report presents the measurement of the $t$-channel cross section [5] and a search for the $W t$-channel production [6]. The $W$ boson from the top-quark decay is always reconstructed in its leptonic decay modes. We report on two measurements based on different techniques to separate signal and background: one employs sequential cuts, the other one is based on a fit to a multivariate discriminant based on a neural network $(\mathrm{NN})$. For the $W t$-channel search the separation of signal and background events is carried out using sequential cuts.

\section{Measurement of single top quark $t$-channel cross section}

Both analyses measuring the $t$-channel cross section reduce the large multijet and $W+$ jets backgrounds using a common selection. Electron candidates are required to have $E_{\mathrm{T}}>25 \mathrm{GeV}$ and $|\eta|<2.47$ and to be well isolated. Muon candidates are reconstructed by combining track segments found in the inner detector and in the muon spectrometer. Muon candidates have to have $p_{T}>25 \mathrm{GeV}$ and $|\eta|<2.5$. Particle jets are reconstructed using the anti- $k_{T}$ algorithm with a width parameter of 0.4. The response of the calorimeter is corrected through a $p_{\mathrm{T}^{-}}$and $\eta$-dependent factor. Only jets having $p_{\mathrm{T}}>25 \mathrm{GeV}$ and $|\eta|<4.5$ are considered. Jets containing bottom quarks are tagged in the region $|\eta|<2.5$ by reconstructing secondary vertices. The final event selection requires exactly one charged lepton, $e$ or $\mu$, exactly two jets or exactly three jets, and missing transverse energy $E_{\mathrm{T}}^{\text {miss }}>25 \mathrm{GeV}$. Since the multijets background is difficult to model precisely its contribution is reduced with $M_{\mathrm{T}}(W)>\left(60 \mathrm{GeV}-E_{\mathrm{T}}^{\text {miss }}\right)$.

The main backgrounds to the single-top quark final state are QCD multijet events, $W$ boson production in association with jets, and top pair production $(t \bar{t})$. Smaller backgrounds and the $t \bar{t}$ background are normalised to their theory predictions. Multijet events contribute as a background to the selected sample if one of the jets in the event is misidentified as an isolated lepton or if the event has a non-prompt lepton that appears isolated. The multijets background normalisation is obtained by a binned maximum likelihood fit to the $E_{\mathrm{T}}^{\text {miss }}$ distribution in the data, using a dataderived template for the multijets background and Monte Carlo templates for all other processes, see Fig. 1a. The kinematic distributions for the $W+$ jets background, that comprises contributions from $W b \bar{b}+$ jets, $W c \bar{c}+$ jets, $W c+$ jets and $W+$ light jets, are taken from Monte Carlo samples, while the overall normalisation and the flavour composition are derived from data. The NN analysis determines the normalization of the different $W+$ jets processes from a simultaneous fit to the NN output distribution when measuring the $t$-channel single top-quark rate, while the cut-based analysis derives scale factors by using the event yields in the 1-jet tag, 2-jet pretag, and 2-jet tag sample, 


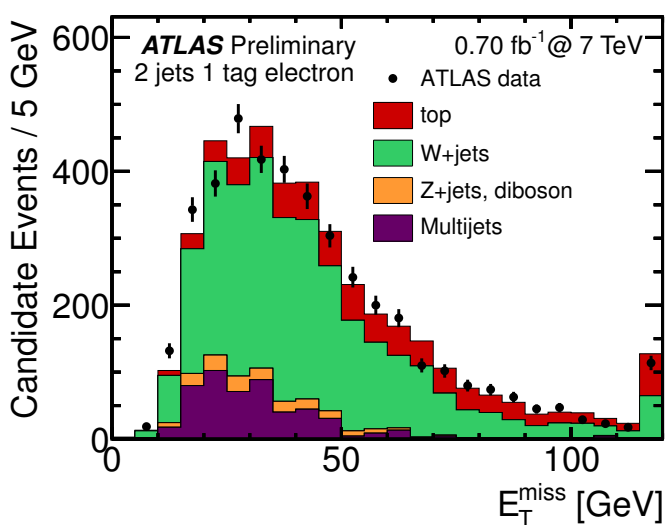

(a)

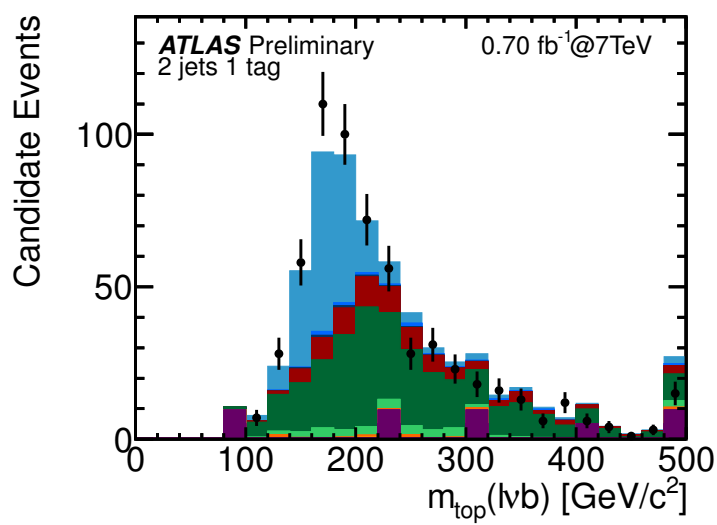

(b)

Figure 1: a) $E_{\mathrm{T}}^{\text {miss }}$ distribution for the 2-jet tag sample. b) Distributions of the reconstructed top quark mass in the 2-jet sample after the jet $\eta, H_{\mathrm{T}}$ and $\Delta \eta$ cuts. The t-channel signal contribution is normalized to the measured combined cut-based cross section [5].

excluding the signal selection. Starting from the preselected samples additional cuts are applied to extract the $t$-channel signal in the cut-based analysis, while the $\mathrm{NN}$ analysis uses all preselected tagged events and constructs a discriminant that separates signal from background events. The cut based analysis uses the following series of cuts to isolate the $t$-channel process: $\mid \eta(1-$ jet $) \mid>2.0$, $H_{\mathrm{T}}>210 \mathrm{GeV}, 150 \mathrm{GeV}<M_{l v b}<190 \mathrm{GeV}$ and $\Delta \eta$ (bjet1, ujet1) $>1.0$. The $M_{l v b}$ distribution is shown after all cuts apart from the cut on $M_{l v b}$ in Fig. 1 b. The neural network analysis combines 13 variables into one discriminant. The most important variables are the reconstructed top-quark mass $M_{l v b}$, the pseudorapidity of the untagged jet $\mid \eta(\mathrm{u}$-jet)|, and the transverse energy of the untagged jet $E_{\mathrm{T}}(\mathrm{u}$-jet $)$. Systematic uncertainties affect the normalisation of the individual backgrounds and the signal acceptance. In the neural network analysis the shape of the individual predictions is also affected; both the rate and the shape uncertainties are taken into account by generating correlated pseudo-experiments. The impact of the systematic uncertainties on the measurement is estimated from these pseudo-experiments. Uncertainties on the object modeling, the Monte Carlo generators, the PDFs, the theoretical cross-sections, the background normalisation to data, and integrated luminosity are considered.

A profile likelihood method was used to estimate the event yields and incorporate the systematic uncertainties in the cut-based analysis. After combining the 2-jet and 3-jet samples the measured cross section and uncertainty is $\sigma_{t}=90_{-9}^{+9}(\text { stat })_{-20}^{+31}$ (syst $)=90_{-22}^{+32} \mathrm{pb}$ with an expected cross section of $\sigma_{t}^{\exp }=65_{-19}^{+28} \mathrm{pb}$. The number of events in each category for the 2 jet bin is shown in Fig. 2a.

To extract the signal content of the selected sample we perform a maximum likelihood fit to the entire NN output distribution. The fitted $t$-channel single top expectation value corresponds to a measured cross section of $\sigma_{t}=105 \pm 7$ (stat $)_{-30}^{+36}$ (syst) $=105_{-31}^{+37} \mathrm{pb}$, the expected measurement being $\sigma_{t}^{\text {exp }}=65 \pm 6(\text { stat })_{-21}^{+28}$ (syst $)=65_{-22}^{+29} \mathrm{pb}$. The output distribution normalized to the fit values is shown in Fig. $2 b$. 


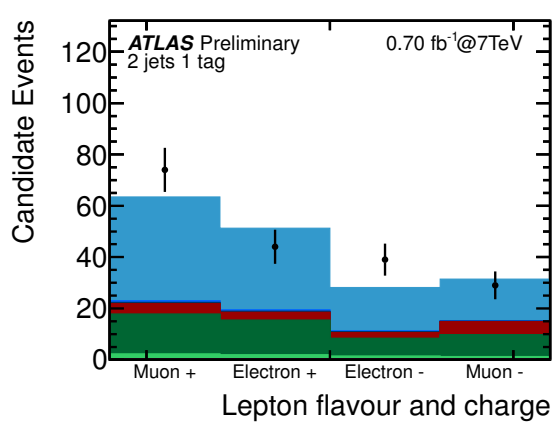

(a)

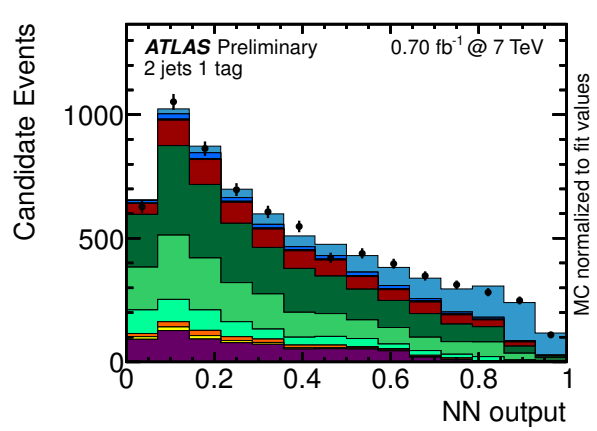

(b)

Figure 2: a) Distribution of the lepton flavour and charge after the cut-based selection in the 2-jet data set. The t-channel signal contribution is normalized to the measured combined cut-based cross section. b) Neural network output distribution normalized to the result of the binned likelihood fit [5].

\section{Search for $W t$ production}

The object definitions used in the search for Wt-production are identical to the $t$-channel analyses. Since we consider only the leptonic decay of both $W$ bosons, exactly two isolated leptons with $p_{\mathrm{T}}>25 \mathrm{GeV}$ and $E_{\mathrm{T}}^{\text {miss }}>50 \mathrm{GeV}$ are required. The signal signature contains a single high- $p_{T}$ jet originating from a $b$ quark and therefore the $p_{\mathrm{T}}$ of the leading jet must be greater than $30 \mathrm{GeV}$. In the dielectron and dimuon channels a cut on the di-lepton mass is required to reduce the large Drell-Yan background $(Z \rightarrow l l)$. In order to reduce the contamination from $Z \rightarrow \tau \tau$ background, a selection is applied on the angle between each lepton and the direction of the $E_{\mathrm{T}}^{\mathrm{miss}}\left(\Delta \phi\left(\vec{l}, E_{\mathrm{T}}^{\overrightarrow{\mathrm{miss}}}\right)\right)$. At this stage, about $85 \%$ of the background originates from the $t \bar{t}$, followed by almost equal contributions from diboson and Drell-Yan events. Signal events are expected to represent $8 \%$ of the preselected sample. Data driven methods are used to estimate the main background contributions, $t \bar{t}$ and Drell-Yan, as well as events selected with fake leptons, see Fig. 3a. The fake lepton and Drell-Yan component of the preselected sample are estimated using data-driven procedures involving side-band regions. The dominant $t \bar{t}$ background is measured directly from data using an orthogonal sample, see Fig. 3b. The signal region is defined by selecting events with exactly one jet, while the $t \bar{t}$ cross-section is measured using preselected events with at least two jets. The $t \bar{t}$ contribution is then extrapolated to the signal sample assuming that the simulation correctly models the $t \bar{t}$ jet multiplicity distribution. A total of $47.1 \pm 3.4 \mathrm{Wt}$ events and of $246 \pm 23$ background events are expected, corresponding to a signal over background ratio of $19 \%$ and a statistical significance of $S / \sqrt{B}=3.0$. The total expected event yield is $293 \pm 23.5$, which is in good agreement with the observed 287 events. The sum of lepton transverse momentum after the final selection is shown in Fig. 3c. The extraction of the $W t$-channel production cross-section is treated as a counting experiment and modeled using a likelihood function. Systematic uncertainties are grouped in uncorrelated sets and their effect is parametrized using a set of nuisance parameters. The cross-section is then obtained by fitting for the maximum likelihood estimate, and the effect of statistical and systematic uncertainties is inferred from the shapes of the likelihood ratio and profile likelihood ratio, respectively. We obtain the following 95\% CL observed upper limit: $\sigma(p p \rightarrow W t+X)<39.1 \mathrm{pb}$, 
while the expected upper limit is : $\sigma(p p \rightarrow W t+X)<40.6 \mathrm{pb}$.

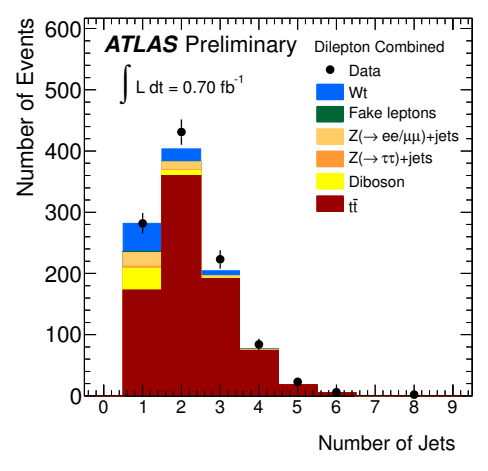

(a)

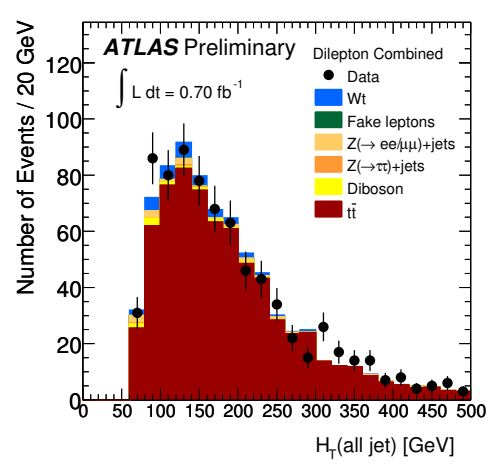

(b)

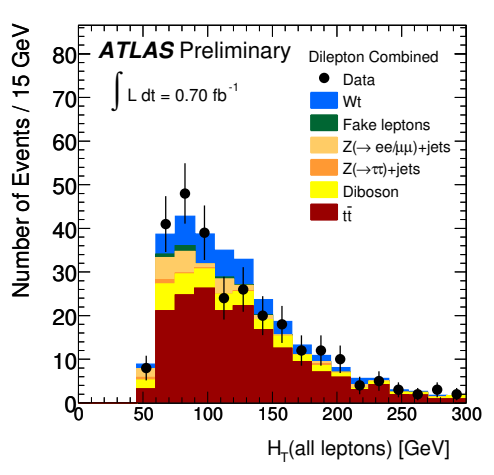

(c)

Figure 3: a) Jet multiplicity distribution for the preselected sample. b) Sum of the jet transverse momentum for events with at least two jets. c) Sum of lepton transverse momentum, shown for 1 -jet events. In b) and c) the $t \bar{t}$ sample is normalized using the scale factor determined in the analysis [6].

\section{Summary}

We have measured the $t$-channel single top-quark production cross section and searched for the associated production of a top quark and a $W$ boson using $0.7 \mathrm{fb}^{-1}$ of data. The $t$-channel single top-quark production cross section was measured using a neural network approach, and a cut based approach. Combining for the later method both 2- and 3-jet channels, the measured cross section is $\sigma_{t}=90_{-9}^{+9}(\text { stat })_{-20}^{+31}$ (syst $)=90_{-22}^{+32} \mathrm{pb}$. with an observed significance of 7.6 standard deviations. The strategy to search for $W t$ channel was performed with a cut-based selection. The $95 \% \mathrm{CL}$ observed limit on $W t$ production is $\sigma(p p \rightarrow W t+X)<39 \mathrm{pb}$.

\section{References}

[1] N. Kidonakis, Next-to-next-to-leading-order collinear and soft gluon corrections for t-channel single top quark production, Phys. Rev. D83 (2011) 091503, arXiv: 1103.2792 [ hep-ph ].

[2] N. Kidonakis, Two-loop soft anomalous dimensions for single top quark associated production with a W- or H-, Phys. Rev. D82 (2010) 054018, arXiv: 1005 . 4451 [ hep-ph ].

[3] N. Kidonakis, NNLL resummation for s-channel single top quark production, Phys. Rev. D81 (2010) 054028, arXiv: 1001.5034 [hep-ph].

[4] T. M. Tait and C.-P. Yuan, Single top quark production as a window to physics beyond the standard model, Phys.Rev. D63 (2000) 014018, arXiv: hep-ph/0007298 [ hep-ph ].

[5] The ATLAS Collaboration, Measurement of the t-channel Single Top-Quark Production Cross Section in $0.70 \mathrm{fb}^{-1}$ of pp Collisions at $\sqrt{(\mathrm{s})}=7 \mathrm{TeV}$ with the ATLAS detector, ATLAS-CONF-2011-101 (2011), http: //cdsweb. cern. ch/record/1369217.

[6] The ATLAS Collaboration, Search for $W+t$ single-top events in the dileptonic channel at ATLAS, ATLAS-CONF-2011-104 (2011), http: / / cdsweb. cern.ch/record/1369829. 